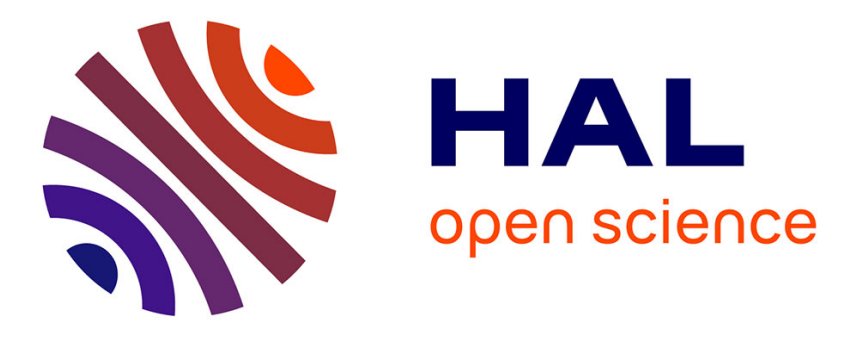

\title{
Spatio-spectral regularization to improve magnetic resonance spectroscopic imaging quantification
}

Andrea Laruelo, Lotfi Chaari, Jean-Yves Tourneret, Hadj Batatia, Soleakhena Ken, Ben Rowland, Régis Ferrand, Anne Laprie

\section{- To cite this version:}

Andrea Laruelo, Lotfi Chaari, Jean-Yves Tourneret, Hadj Batatia, Soleakhena Ken, et al.. Spatiospectral regularization to improve magnetic resonance spectroscopic imaging quantification. NMR in Biomedicine, 2016, 129 (7), pp.918-931. 10.1002/nbm.3532 . hal-01381730

\author{
HAL Id: hal-01381730 \\ https://hal.science/hal-01381730
}

Submitted on 14 Oct 2016

HAL is a multi-disciplinary open access archive for the deposit and dissemination of scientific research documents, whether they are published or not. The documents may come from teaching and research institutions in France or abroad, or from public or private research centers.
L'archive ouverte pluridisciplinaire HAL, est destinée au dépôt et à la diffusion de documents scientifiques de niveau recherche, publiés ou non, émanant des établissements d'enseignement et de recherche français ou étrangers, des laboratoires publics ou privés. 


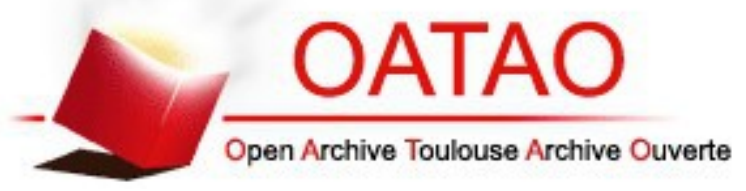

\section{Open Archive TOULOUSE Archive Ouverte (OATAO)}

OATAO is an open access repository that collects the work of Toulouse researchers and makes it freely available over the web where possible.

This is an author-deposited version published in : http://oatao.univ-toulouse.fr/ Eprints ID : 16139

To link to this article : DOI:10.1002/nbm.3532

URL : http://dx.doi.org/10.1002/nbm.3532

To cite this version : Laruelo, Andrea and Chaari, Lotfi and Tourneret, Jean-Yves and Batatia, Hadj and Ken, Soleakhena and Rowland, Ben and Ferrand, Régis and Laprie, Anne Spatio-spectral regularization to improve magnetic resonance spectroscopic imaging quantification. (2016) NMR in Biomedicine, vol. 129 (n 7). pp. 918931. ISSN 0952-3480

Any correspondence concerning this service should be sent to the repository administrator: staff-oatao@,listes-diff.inp-toulouse.fr 


\title{
Spatio-spectral regularization to improve magnetic resonance spectroscopic imaging quantification
}

\author{
Andrea Laruelo ${ }^{a, b *}$, Lotfi Chaari ${ }^{\mathbf{b}, c}$, Jean-Yves Tourneret ${ }^{\mathbf{b}}$, Hadj Batatiab, \\ Soléakhéna Ken ${ }^{\mathrm{a}, \mathrm{e}}$, Ben Rowland ${ }^{\mathrm{a}}$, Régis Ferrand ${ }^{\mathrm{a}}$ and Anne Laprie ${ }^{\mathrm{a}, \mathrm{d}, \mathrm{e}}$
}

\begin{abstract}
Magnetic resonance spectroscopic imaging (MRSI) is a non-invasive technique able to provide the spatial distribution of relevant biochemical compounds commonly used as biomarkers of disease. Information provided by MRSI can be used as a valuable insight for the diagnosis, treatment and follow-up of several diseases such as cancer or neurological disorders. Obtaining accurate metabolite concentrations from in vivo MRSI signals is a crucial requirement for the clinical utility of this technique. Despite the numerous publications on the topic, accurate quantification is still a challenging problem due to the low signal-to-noise ratio of the data, overlap of spectral lines and the presence of nuisance components. We propose a novel quantification method, which alleviates these limitations by exploiting a spatio-spectral regularization scheme. In contrast to previous methods, the regularization terms are not expressed directly on the parameters being sought, but on appropriate transformed domains. In order to quantify all signals simultaneously in the MRSI grid, while introducing prior information, a fast proximal optimization algorithm is proposed. Experiments on synthetic MRSI data demonstrate that the error in the estimated metabolite concentrations is reduced by a mean of $41 \%$ with the proposed scheme. Results on in vivo brain MRSI data show the benefit of the proposed approach, which is able to fit overlapping peaks correctly and to capture metabolites that are missed by single-voxel methods due to their lower concentrations.
\end{abstract}

\section{INTRODUCTION}

Magnetic resonance spectroscopic imaging (MRSI) is a noninvasive technique able to provide relevant information on tumour characteristics, progression and response to treatment not available from conventional morphological magnetic resonance imaging (MRI) $(1,2)$. It has become a valuable tool for the examination of human brain tumours, prostate and breast cancers and several neurological disorders $(3,4)$. Despite the numerous publications on the subject (5-11), the quantification of MRSI data is still a challenging problem, due to the low signalto-noise ratio (SNR) of MRSI signals and the overlap of spectral lines. The SNR and chemical shift separation of metabolite peaks increase approximately linearly with the increase of magnetic field.

\footnotetext{
* Correspondence to: A. Laruelo, Institut Claudius Regaud, Institut Universitaire du Cancer de Toulouse-Oncopole, 1 Avenue Irène Joliot Curie, F-31059 Toulouse, France. E-mail: andrea.laruelo@gmail.com

a A. Laruelo, S. Ken, B. Rowland, R. Ferrand, A. Laprie Institut Claudius Regaud, Institut Universitaire du Cancer de ToulouseOncopole, Toulouse, France

b A. Laruelo, L. Chaari, J.-Y. Tourneret, H. Batatia

University of Toulouse, IRIT - INP-ENSEEIHT, Toulouse, France

C L. Chaari

MIRACL Laboratory, Sfax, Tunisia

d A. Laprie

Université Toulouse III Paul Sabatier, Toulouse, France
}

However, considering the relatively weak magnetic fields used in clinical practice, a limited chemical shift dispersion often causes spectral overlap and complicates the separation of metabolites. The difficulty is higher when there are lesions that produce noncharacteristic spectra and when nuisance signals, such as poorly suppressed water or lipid signals in ${ }^{1} \mathrm{H}-\mathrm{MRSI}$, distort the spectra and desirable metabolite signals are almost indistinguishable from noise due to their low concentration. Therefore, improving the SNR is a key factor in achieving robust and accurate quantification of MRSI data. The SNR can be improved by increasing the signal using higher magnetic field strength. However, the implementation of MRSI at high fields (7 Tesla ( $\mathrm{T}$ ) and beyond) is still challenging, owing to technical limitations that may reduce the expected SNR, such as increased field inhomogeneity (12). In fact, currently clinical

\author{
e S. Ken, A. Laprie \\ INSERM UMR1214 TONIC, Toulouse, France
}

Abbreviations used: AMARES, advanced method for accurate robust and efficient spectral fitting; AQSES, automated quantitation of short echo time MRS spectra; Cho, choline; CSI, chemical shift imaging; $C r$, creatine; CRLB, CramérRao lower bound; $d B$, decibel; FID, free induction decay; FOV, field of view; HSVD, Hankel singular-value decomposition; jMRUI, Java-based graphical user interface for processing MRS; Lac, lactate; LCModel, quantification approach proposed in (7); MR, magnetic resonance; NAA, N-acteyl-aspartate; PPM, parts per million; PRESS, point-resolved spectroscopy; QUEST, quantitation based on semiparametric quantum estimation; RMSE, root-mean-squared error; SNR, signal-tonoise ratio; SSIM, structural similarity index; SSR, spatial-spectral regularization; $T$, Tesla, ; TE, echo time; TR, repetition time. 
manufacturers do not support field strengths higher than $3 \mathrm{~T}$. Signal averaging is a widely used technique to improve the SNR at acquisition time. However, this approach is not feasible because of long acquisition times. Moreover, physiological motion may lead to destructive averaging, due to variations in signal phase and frequency. Methods to increase the SNR at the processing level have also been proposed (13-15). Unfortunately, most of these methods rely heavily on spectral constraints, limiting their ability to capture the spatial variations exhibited by in vivo scans, especially in the context of lesions. As a consequence, they are often ineffective in practical applications (16).

Many quantification methods also incorporate techniques to address the low quality of MRSI signals. A common approach to improve the robustness of the quantification of MRSI spectra is the incorporation of prior knowledge for some spectral parameters. For example, AMARES (5) allows specification of upper and lower bounds for frequency, damping and phase. It also allows imposition of relations (such as differences or ratios) between individual spectral parameters. Simulations as well as in vivo experiments confirm the decrease of the Cramér-Rao lower bound (CRLB) (17) when incorporating prior knowledge in the quantification process (18). Prior knowledge of the spectral parameters can also be incorporated by making use of experimentally measured (in vitro) or simulated metabolite profiles $(19,20)$, as implemented in (7-9). More recently, some quantification methods have proposed exploiting not only the spectral but also the spatial context of MRSI signals by incorporating spatial priors into the quantification model. An early approach using $a$ priori spatial information was introduced by Soher et al. (6). In their iterative method, selected parameter estimates are updated at each iteration assuming the smoothness of these parameter distributions. The well-known LCModel (7) also provides a spatial fitting mode for MRSI data. It first analyzes a central voxel and then proceeds in the neighbuorhood using previously fitted voxels for initialization and soft constraints for new fits. In (11), a Bayesian smoothness prior improving the fitting of MRSI data is proposed. This method encourages spatially smooth variations for some selected spectral parameters (frequency, damping and phase). In (10), an iterative approach combining the spatial prior proposed in (11) with other methods incorporating spatial information is presented. Spatial smoothing is desirable, because it is reasonable to expect that neighbouring pixels will contain similar information. However, it is well known that spatial smoothing tends to blur the images, since the edges between different regions may be smoothed out.

In this article, we propose a novel quantification technique that benefits from both model-based and regularized quantification methods. Spatial and spectral regularization terms are incorporated jointly, with the aim of increasing the SNR and exploiting the neighbouring information along the spatial and spectral dimensions for a given MRSI voxel. In contrast to previous methods, this prior knowledge is not imposed directly on selected spectral parameters but rather on the representation of the data in the wavelet domain. The method exploits the fact that the wavelet transform maps white noise in the signal domain to white noise in the transform domain. Thus, while signal energy becomes more concentrated into fewer coefficients in the wavelet domain, noise energy does not. This property enables the efficient separation of signal from noise. A number of wavelet-based techniques, including quantification and denoising, have previously been applied to magnetic resonance (MR) spectroscopy (21-24). Fast MR spectroscopic imaging has also been accomplished by the use of wavelets to enable compression in the spectral and spatial domains $(25,26)$. To our knowledge, this is the first time that the use of the wavelet properties together with a spatio-spectral regularization has been integrated into the quantification algorithm. The hybrid regularization is able to catch the different nature of the spectral and spatial dimensions of MRSI data. It is worth noting that the proposed method can be applied in addition to other types of prior knowledge (such as the ones mentioned previously). A quantification solution is formulated for the whole MRSI grid. However, in contrast to previous approaches, the presented method is more flexible and less restrictive, allowing the preservation of sharp spatial features. In order to fit all signals in the MRSI grid simultaneously while introducing spectral-spatial information, a fast proximal optimization algorithm is proposed to recover the optimal solution.

\section{THEORY}

\section{Signal model}

In vivo MRS signals are measured in the time domain and consist of responses from metabolites (including macromolecules), water and noise. Theoretically, a measured MRS signal at a spatial position $r \in\{1, \ldots, R\}$ can be modelled by a sum of $K$ exponentially damped complex sinusoids (27) of $M$ time-domain data samples:

$$
\begin{aligned}
s^{r}(m)= & \sum_{k=1}^{K} a_{k}^{r} \exp \left(i \phi_{k}\right) \exp \left(-d_{k} m+2 \pi i f_{k} m\right) \\
& +\boldsymbol{b}^{r}(m)+\boldsymbol{w}^{r}(m)+\boldsymbol{n}^{r}(m)
\end{aligned}
$$

where $m=1, \ldots, M$. In (1), the amplitude $a_{k}^{r}$ is proportional to the number of nuclei contributing to the spectral component of frequency $f_{k}$. The damping factor $d_{k}$ provides information about the mobility and macromolecular environment of the nucleus, $\varphi_{k}$ is the phase, $\boldsymbol{b}^{r}$ is the baseline, i.e. the contribution from macromolecules, $\boldsymbol{w}^{r}$ denotes the water signal and the noise term $\boldsymbol{n}^{r}$ is assumed to be a circular complex white Gaussian noise.

Recent quantification algorithms impose spectral prior knowledge using metabolite templates (7-9). The MRS signal can therefore be described as a linear combination of basis functions, leading to

$$
\boldsymbol{s}^{r}(m)=\sum_{k=1}^{K} a_{k}^{r} \boldsymbol{h}_{k}(m)+\boldsymbol{b}^{r}(m)+\boldsymbol{w}^{r}(m)+\boldsymbol{n}^{r}(m)
$$

where $\boldsymbol{h}_{k}$ denotes a basis function (in the time or frequency domain) and the amplitudes $a_{k}^{r}$ are proportional to the concentration of the respective metabolites. Note that $m$ can refer to either a temporal or spectral index in (2). The following subsection introduces the new quantification model investigated in this study.

\section{Quantification model}

In MRS, quantification is the estimation of spectral parameters from measured data. Signal amplitude (in the time domain) is 
the main parameter of interest, since it is proportional to the metabolite concentration. Let $\mathbf{S} \in \mathbb{C}^{M \times R}$ be the observed MRSI signal corresponding to a two-dimensional (2D) slice involving $M$ spectral points and $R$ spatial positions. Also let $\boldsymbol{A} \in \mathbb{C}^{K \times R}$ be the matrix containing the contribution of the $K$ metabolites to the observed signal at each spatial position $r=1, \ldots, R$. According to (2) and assuming that the water signal and the macromolecular contribution have been previously filtered, we propose to estimate the metabolite amplitudes from the following inverse problem:

$$
\mathbf{S}=\boldsymbol{H A}+\boldsymbol{N}
$$

where

$$
\begin{gathered}
H \triangleq\left[\begin{array}{ccc}
h_{1}(1) & \ldots & h_{K}(1) \\
\vdots & \vdots & \vdots \\
h_{1}(M) & \ldots & h_{K}(M)
\end{array}\right], \quad A \triangleq\left[\begin{array}{ccc}
a^{1}(1) & \ldots & a^{R}(1) \\
\vdots & \vdots & \vdots \\
a^{1}(K) & \ldots & a^{R}(K)
\end{array}\right] \\
S \triangleq\left[\begin{array}{ccc}
s^{1}(1) & \ldots & s^{R}(1) \\
\vdots & \vdots & \vdots \\
s^{1}(M) & \ldots & s^{R}(M)
\end{array}\right], \text { and } N \triangleq\left[\begin{array}{ccc}
n^{1}(1) & \ldots & n^{R}(1) \\
\vdots & \vdots & \vdots \\
n^{1}(M) & \ldots & n^{R}(M)
\end{array}\right]
\end{gathered}
$$

For each $k=1, \ldots, K, \boldsymbol{h}_{k}=\left[h_{k}(1), \ldots, h_{k}(M)\right]^{\top}$ denotes an element of the metabolite basis (spectral profile). For a fixed spatial position $r, \boldsymbol{a}^{r}=\left[a^{r}(1), \ldots, a^{r}(K)\right]^{\top}$ is a column vector containing the contributions of the different metabolites to the observed MRS signal and $\boldsymbol{n}^{r}=\left[n^{r}(1), \ldots, n^{r}(M)\right]^{\top}$ is an additive complex-valued Gaussian noise of unknown covariance matrix $\Psi$. Note here that the spatial positions have been vectorized so that a $2 \mathrm{D}$ slice is represented by an $R$-dimensional vector.

\section{METHODS}

\section{Classic resolution scheme}

Our goal here is to solve the inverse problem (3) in order to obtain an estimate $\hat{A}$ based on the observation $\boldsymbol{S}$, the knowledge of the basis $\boldsymbol{H}$ and the noise covariance matrix $\boldsymbol{\Psi}$. A basic solution to this problem is based on the minimization of the weighted least-squares criterion:

$$
\hat{\boldsymbol{A}}_{\mathrm{WLS}}=\arg \min _{\boldsymbol{A}} \mathcal{J}_{\mathrm{WLS}}(\boldsymbol{A})=\arg \min _{\boldsymbol{A}}\|\boldsymbol{H} \boldsymbol{A}-\boldsymbol{S}\|_{\boldsymbol{\Psi}}^{2}
$$

which leads to

$$
\hat{\boldsymbol{A}}_{\mathrm{WLS}}=\left(\boldsymbol{H}^{H} \boldsymbol{\Psi}^{-1} \boldsymbol{H}\right)^{\#} \boldsymbol{H}^{H} \boldsymbol{\Psi}^{-1} \boldsymbol{S}
$$

where $(\cdot)^{H}$ stands for the transposed complex conjugate, $(\cdot)^{\#}$ stands for the pseudo-inverse and $\|\cdot\|_{\boldsymbol{\Psi}^{-1}}=\sqrt{(\cdot)^{\mathrm{H}} \boldsymbol{\Psi}^{-1}(\cdot)}$ defines a norm on $\mathbb{C}^{K}$.

Due to the instability of the covariance matrix or the illconditioning of the metabolite basis $\boldsymbol{H}$, the inverse problem (3) is generally ill-posed. In this case, the solution $\hat{\boldsymbol{A}}_{\text {WLS }}$ can be unstable and of poor quality. For this reason, one must generally resort to regularization (28). Regularization simply consists of introducing some prior knowledge on the target solution. This issue has been widely investigated in the MRI literature during the last decade (29-32). Different priors have therefore been proposed promoting smoothness or sparsity of the target solutions, either in the original space or in a transformed domain.

\section{Spatio-spectral regularization}

To the best of our knowledge, all existing methods for regularized MRSI quantitation incorporate spatial smoothness assumptions on some spectral parameters such as phase or line width $(10,11)$. While promoting spatial smoothness for such specific parameters, no constraint is used for the signal amplitudes. In this article, we propose to incorporate prior knowledge on the MRSI data using a different regularization approach. On one hand, we exploit the sparsity of MR spectra by constraining the $\ell_{1}$ norm of the wavelet transform. On the other hand, we favour solutions (fits) with few irregularities in the spatial dimension by penalizing the wavelet coefficients of the MRSI grid at each frequency band (Fig. 1). The term 'irregularities' mostly refers to the noise and nuisance components typically present in MRSI data. In fact, in MRSI-acquisition conditions, the noise level is often similar in magnitude to some metabolite signal levels (like lactate) and noise interferes with signal identification and determination of the metabolite amplitudes.

MRSI data might not be strictly sparse with respect to the $\ell_{0}$ pseudo-norm, due to the typically low spatial resolution of this type of data. However, we aim to exploit the fact that MRSI data can be represented using only a few dominating coefficients in the wavelet domain, leading to a low $\ell_{1}$ norm. This is true for both spectral and spatial dimensions of the data. Indeed, the spatio-spectral sparsity of MRSI data has already been used successfully in compressed sensing imaging approaches $(25,26)$.

This prior knowledge is included in the inversion scheme by using two regularization terms in the optimality criterion $\mathcal{J}_{\text {wLS }}$ in (5). Note that the proposed approach can be easily adapted to any convex penalization in both spectral and spatial dimensions.

\section{Regularization terms}

At a given spectral frequency $m$, let us denote by $(\boldsymbol{H} \boldsymbol{A})^{m} \in \mathbb{C}^{R}$ the 2D complex-valued image of size $R=N_{r} \times N_{c}$ that results from reshaping the $m$ th row ( $R$-dimensional vector) of the matrix $\boldsymbol{H A}$. We employ a dyadic 2D orthonormal wavelet decomposition operator $T$ over $J^{1}$ resolution levels. The coefficient field resulting from the wavelet decomposition of a target image $(\boldsymbol{H} \boldsymbol{A})^{m}$ is defined as $\zeta^{m}=\left(\zeta_{a}^{m},\left(\zeta_{o, j}^{m}\right)_{o \in \mathbb{O}, 1 \leq j \leq J^{\prime}}\right)$, with $\quad o \in \mathbb{O}=$ $\{0,1\}^{2} \backslash\{(0,, 0)\}, \zeta_{a}^{m}=\left(\zeta_{a, l}^{m}\right)_{1 \leq l \leq L_{j 1}}$ and $\zeta_{o, j}^{m}=\left(\zeta_{o, j, l}^{m}\right)_{1 \leq I \leq L_{j}}$, where $L_{j}=R \times 2^{-2 j}$ is the number of wavelet coefficients in a given sub-band at resolution $j$ (by assuming that $N_{r}$ and $N_{c}$ are multiples of $2^{J^{1}}$ ). Adopting such a notation, the wavelet coefficients can be re-indexed so that $\zeta_{a}^{m}$ denotes the approximation coefficient vector at the resolution level $J^{1}$, while $\zeta_{o . j}^{m}$ denotes the detail coefficient vector at orientation $o$ and resolution level $j$.

On the other hand, let us denote by $(\boldsymbol{H} \boldsymbol{A})^{r} \in \mathbb{C}^{M}$ the $r$ th column of the matrix $M$ corresponding to the spectrum of size $M$ 

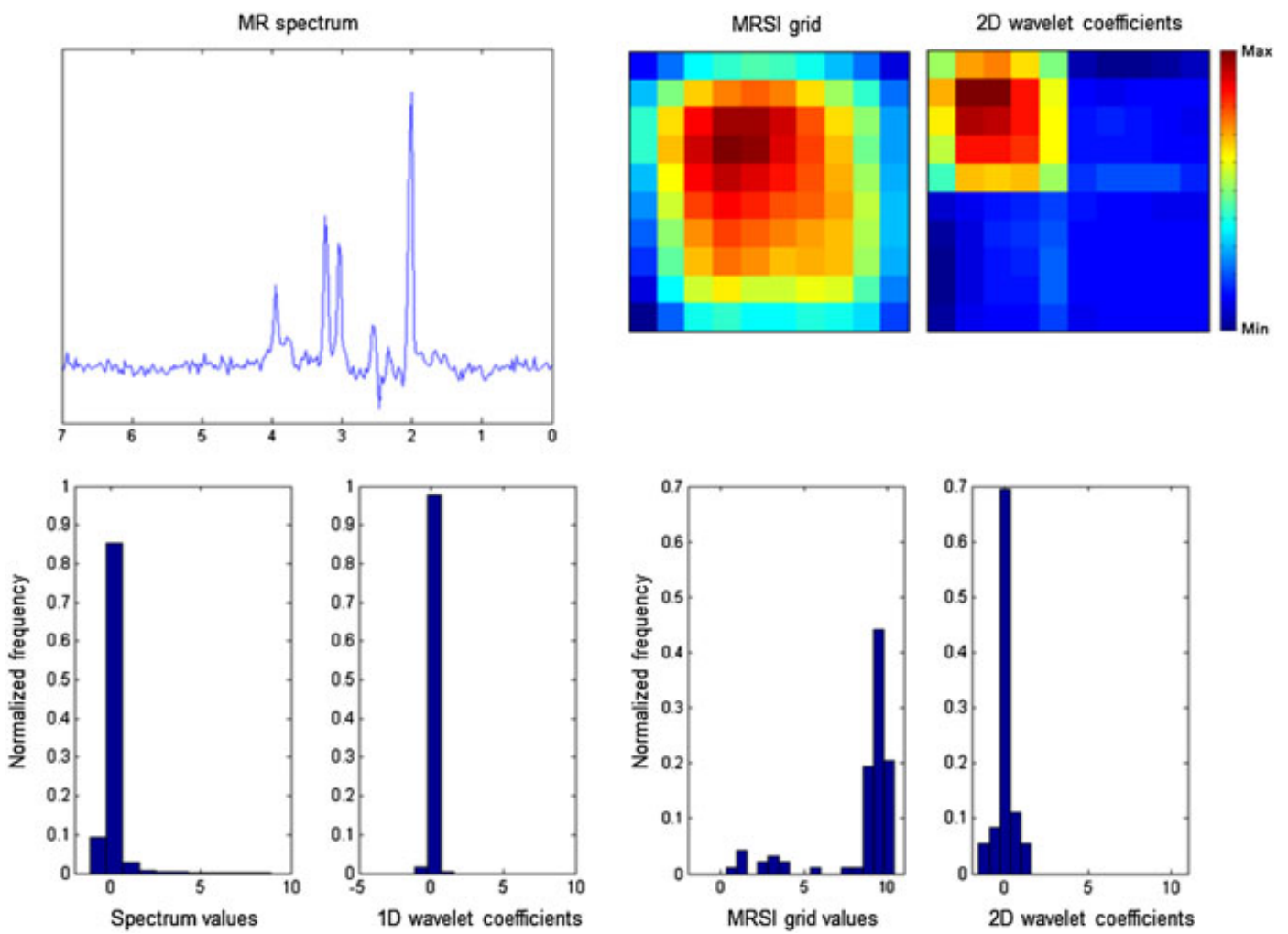

Figure 1. MRSI data have a few dominating coefficients in the wavelet domain in the spectral and spatial dimensions. Top left: real part of a MR spectrum. Top right: MRSI data set at a specific frequency position (MRSI grid) and its corresponding 2D wavelet transform. Bottom left: normalized histograms showing the values of a MR spectrum $\left(\ell_{0}\right.$ norm) and its corresponding 1D wavelet coefficients. Bottom right: normalized histograms showing the values of a MRSI grid (at a specific frequency position)and its corresponding 2D wavelet coefficients. Only the detail wavelet coefficients are shown in both cases.

at spatial position $r$. We denote here by $F$ the dyadic 1D orthonormal wavelet decomposition operator over $\mathcal{J}^{2}$ resolution levels. The coefficient field corresponding to the spectrum $(\boldsymbol{H} \boldsymbol{A})^{r}$ is then denoted by $\zeta^{r}=\left(\begin{array}{l}r \\ a\end{array},\left(\begin{array}{l}r \\ d, j\end{array}\right)_{1 \leq j \leq J^{2}}\right)$, where the subscripts $a$ and $d$ denote approximation and detail subbands, respectively.

In order to constrain the solution $\hat{A}$ to have few irregularities in both spatial and spectral dimensions, we propose to introduce two regularization terms. The first one describes the $2 \mathrm{D}$ spatial prior knowledge about the wavelet coefficients of the target solution. This first regularization term is defined as

$$
\begin{aligned}
g_{1}(\boldsymbol{A})= & \sum_{m=1}^{M}\left\{\sum_{l=1}^{L_{j 1}} \Phi_{\mu_{a}, \alpha_{a}}^{p}\left[\left(T(\boldsymbol{H} \boldsymbol{A})^{m}\right)_{a, l}\right]\right. \\
& \left.+\sum_{o \in \mathbb{O}} \sum_{j=1}^{J^{1}} \sum_{l=1}^{L_{j}} \Phi_{\mu_{o}^{j}, \alpha_{o}^{j}}^{p}\left[\left(T(\boldsymbol{H} \boldsymbol{A})^{m}\right)_{o, j, l}\right]\right\}
\end{aligned}
$$

where $\Phi_{\mu, \alpha}^{p}(\xi)=\alpha|\xi-\mu|_{p^{\prime}}^{p} \forall \xi \in \mathbb{C}, \mu_{o}^{j} \in \mathbb{C}_{,} \alpha_{o}^{j} \in \mathbb{R}_{+}$and $p \geq 1$ (and similarly for $\mu_{a}$ and $\alpha_{a}$ ). Due to the shape parameter $p$, this regularization term makes a compromise between sparsity $(p \sim 1)$ and smoothness $(p \sim 2)$ of the wavelet coefficients of the 2D image $(\boldsymbol{H} \boldsymbol{A})^{m}$. The second regularization term allows us to reduce high spectral discontinuities (especially close to the metabolite peaks) between adjacent frequency bands at a voxel $r$. This regularization is made through the penalization of the wavelet coefficients of the 1D spectra $(\boldsymbol{H A})^{r}$ :

$$
\begin{aligned}
g_{2}(\boldsymbol{A})= & \sum_{r=1}^{R}\left\{\sum_{l=1}^{L_{j 2}} \Phi_{\eta_{a}, \lambda_{a}}^{\beta}\left[\left(F(\boldsymbol{H} \boldsymbol{A})^{r}\right)_{a, l}\right]\right. \\
& +\sum_{j=1}^{\rho^{2}} \sum_{l=1}^{L_{j}} \Phi_{\eta_{d}^{j}, \lambda_{d}^{j}}^{\beta}\left[\left(F(\boldsymbol{H A})^{r}\right)_{d, j, k}\right\}
\end{aligned}
$$

where $\eta_{d}^{j} \in \mathbb{C}, \lambda_{d}^{j} \in \mathbb{R}_{+}$and $\beta \geq 1$ (similarly for $\eta_{a}$ and $\lambda_{a}$ ).

Note that this kind of $\ell_{p}$ regularization has already been used successfully in sparse MRI reconstruction $(31,32)$.

\section{Optimization procedure}

Based on the spatial and spectral regularization terms defined in (7) and (8) (resp. $g_{1}(\boldsymbol{A})$ and $g_{2}(\boldsymbol{A})$ ) and accounting for the quadratic data fidelity term, $\mathcal{J}_{\text {WLS }}$, the criterion to be optimized can be written as

$$
\mathcal{J}(\boldsymbol{A})=\mathcal{J}_{\mathrm{WLS}}(\boldsymbol{A})+g_{1}(\boldsymbol{A})+g_{2}(\boldsymbol{A})
$$

Note here that if, for instance, one wants to perform only spatial regularization, the spectral regularization term can be removed by setting $\lambda_{d}^{j}=\lambda_{a}=0$ in (8). However, using both regularizations allows high spectral and spatial discontinuities to be eliminated.

Since $\mathcal{J}$ is strictly convex, the uniqueness of its minimizer is guaranteed. However, $\mathcal{J}$ is not necessarily differentiable, which makes the use of standard gradient-based algorithms for minimization impossible. We therefore propose to perform the 
minimization of $\mathcal{J}$ in (9) by using the concept of proximity operators (33), which was found to be fruitful in a number of recent works on convex optimization $(31,34)$.

Since the cost function in (9) is made up of more than two non-necessarily differentiable terms, an appropriate solution for its minimization is the simultaneous direction method of multipliers algorithm (34). A key advantage of this proximal algorithm is that it can be parallelized while converging to the global minimum. More details about the optimization procedure using a proximal algorithm can be found in the Appendix.

\section{MATERIAL}

\section{Experiments for synthetic data}

In order to evaluate the performance of the proposed method, different experiments on synthetic data were designed. Simulated MRSI data sets were constructed by combining profiles from the principal metabolites observed in long echo time brain MRS: choline (Cho), creatine (Cr), N-acetyl-aspartate (NAA) and lactate (Lac). Metabolite profiles were obtained from quantum mechanical simulations of a spin-echo MR experiment using the jMRUI software package (35). First, $N=50$ Monte Carlo simulations of MRSI data sets of size $10 \times 10$ were performed to measure the accuracy of the proposed method as a function of SNR. To this aim, five different levels of white Gaussian noise were added to a noiseless simulated data set. The SNR in dB of each signal, $\boldsymbol{S}$, was computed in the frequency domain as $S N R=20 \log _{10}\left\|\boldsymbol{S}_{\text {ref }}\right\|_{2} /\left(\left\|\boldsymbol{S}_{\text {ref }}-\boldsymbol{S}\right\|_{2}\right)$, where $\boldsymbol{S}_{\text {ref }}$ denotes the noiseless signal. Figure 2 shows examples of spectra for different noise levels considered in this experiment.

The root-mean-squared error (RMSE) of the estimated amplitudes for each metabolite $k$ was calculated for each voxel $r$ as

$$
\operatorname{RMSE}_{k, r}=\sqrt{\frac{1}{N} \sum_{n=1}^{N} \frac{\left(a_{k, r, n}^{\mathrm{ref}}-\widehat{a}_{k, r, n}\right)^{2}}{\left(a_{k, r, n}^{\mathrm{ref}}\right)^{2}}}
$$

where $a_{k, r, n}^{\text {ref }}, \hat{a}_{k, r, n}$ are the true and estimated amplitudes of the $k$ th metabolite at voxel $r$ for the $n$th simulation, respectively.

For each noise level, the performance of the proposed method is compared with the performance from a voxel-wise quantification method (AQSES (9)) and from AQSES-MRSI (the extension of AQSES for MRSI data $(10,36))$. In order to provide an indication of the gain in accuracy provided by the proposed method, the corresponding Cramér-Rao lower bounds (17) of the single-voxel approach are also shown.

A second experiment was considered in order to check that the proposed method preserves spatial features. Two MRSI data sets of size $10 \times 10$ representing different spatial features were generated. Both data sets contain a region with healthyappearing signals and a region with tumour-like signals. In the first case there is a sharp transition between the two regions, while a smoother change is applied for the second data set. The structural similarity index (SSIM) (37) was estimated for the amplitude map of each metabolite. This index provides a valuable measure of performance over the whole grid for each metabolite.

For all experiments (for both synthetic and in vivo data), a Daubechies basis with two vanishing moments over one resolution level $\left(J^{1}=1\right.$ and $J^{2}=1$ in (7) and (8), respectively) was considered. Different types of wavelet families were tested and Daubechies was shown to be the best suited for this type of data. The reason to use one resolution level is twofold. On one hand, no significant improvements were noticed using resolution levels greater than one (16). In the spatial dimension, this is due mainly to the typically low spatial resolution of the MRSI images used in the clinical routine (no bigger than $32 \times 32$ ). On
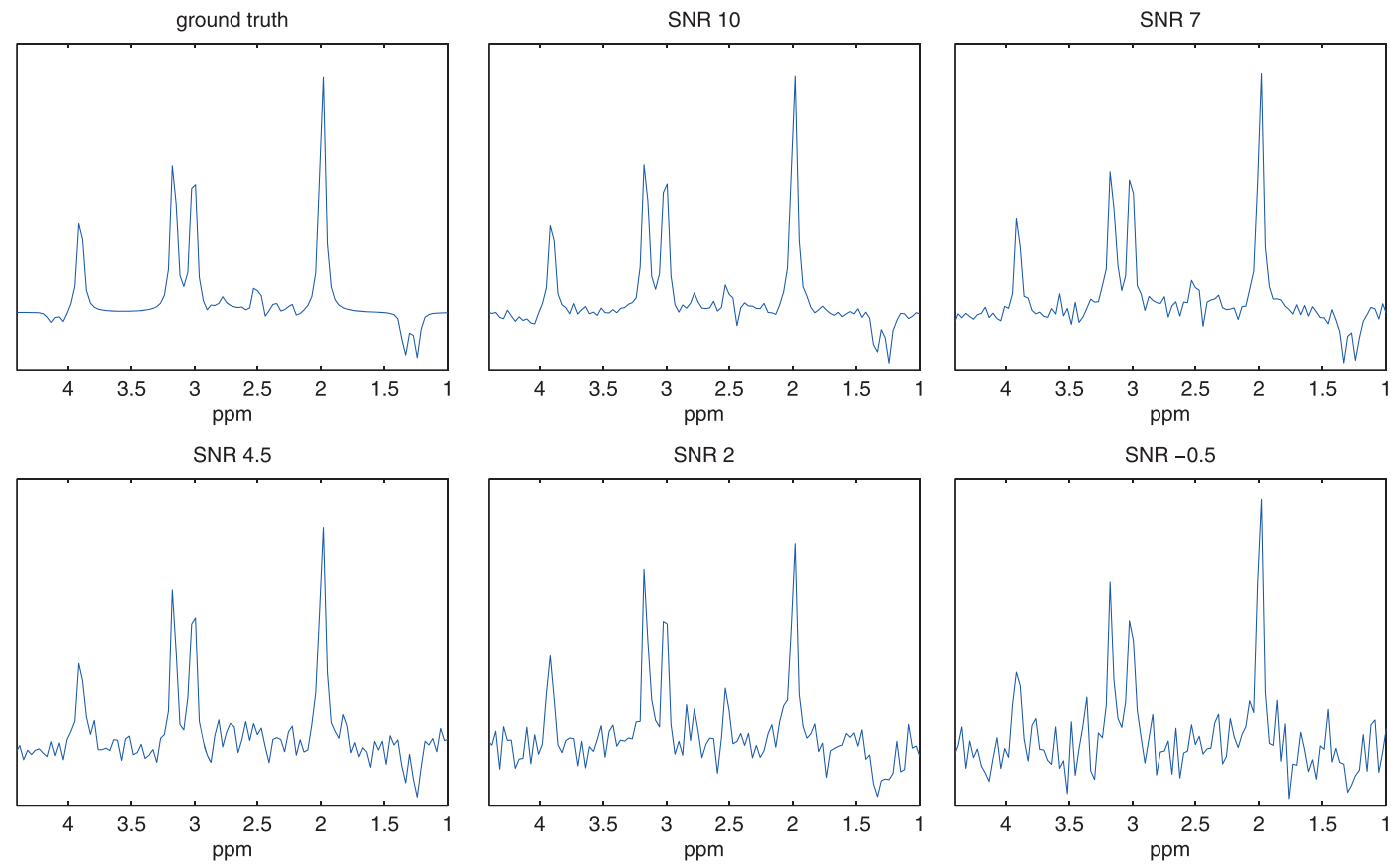

Figure 2. Synthetic MRSI spectra for different noise levels [SNR $=-0.5,2,4.5,7,10]$ and noiseless ground truth. 
the other hand, increasing the number of resolution levels increases the number of regularization parameters to be set, which could lead to less preciseness in the prior information used for regularization.

In order to promote sparse solutions in the wavelet domain, the values of $p$ and $\beta$ in equations (7) and (8), respectively, are set to $p=\beta=1$ for all experiments. Note that only detail coefficients were processed. This is equivalent to setting the regularization parameter to zero for the approximation coefficients. Regularization parameters were selected manually by testing a number of values for each MRSI data set. The combination of parameters providing the best trade-off between spectral SNR and spatial features preservation was selected. It is worth noticing that fully autocalibrated approaches may be used to estimate these parameters automatically directly from the data $(38,39)$.

\section{Experiments for in vivo data}

In vivo MRSI data were acquired at Institut Claudius Regaud using a 1.5 T clinical MR scanner (Siemens Magnetom Avanto) using a 3D chemical shift imaging (CSI) sequence with water suppression, $\mathrm{TE}=135 \mathrm{~ms}$, TR =1500 ms, 512 free induction decay (FID) data points, 4 averages, slice thickness $15 \mathrm{~mm}$, matrix size $16 \times 16$, field of view (FOV) $160 \times 160 \mathrm{~mm}$, yielding a voxel size of $10 \mathrm{~mm} \times 10 \mathrm{~mm} \times 15 \mathrm{~mm}$. Data were acquired from a healthy

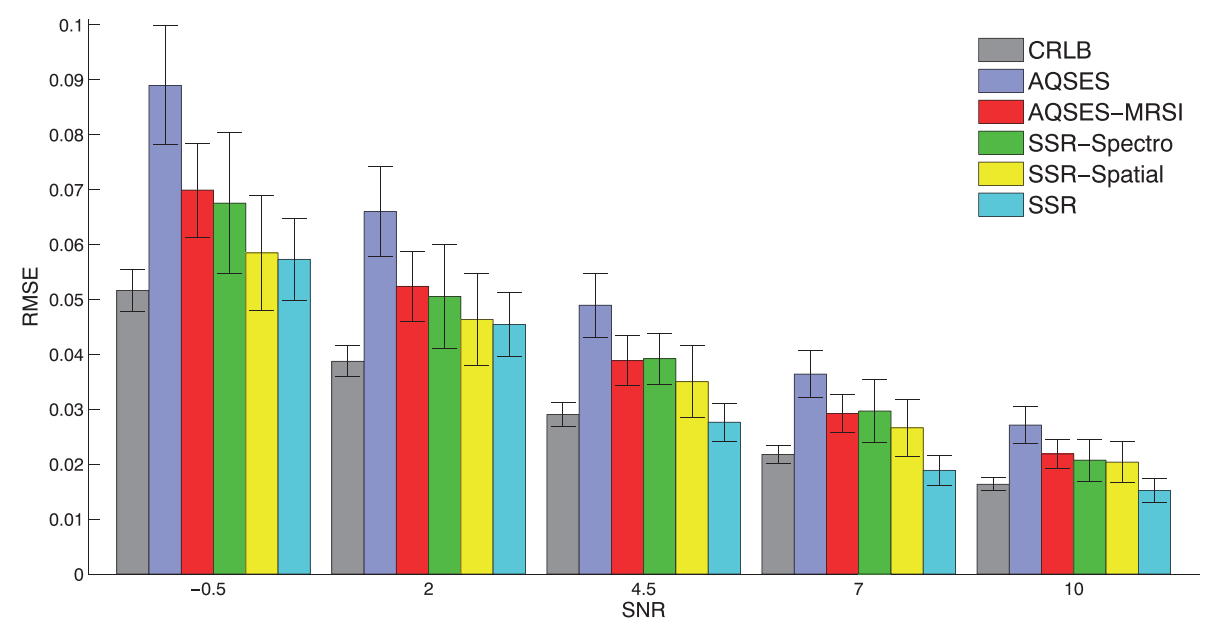

Figure 3. RMSE $E_{k, r}$ of the estimated metabolite amplitudes averaged over all metabolites and all voxels computed for 50 synthetic MRSI data sets of size $10 \times 10$ for five different noise levels (SNR values are: $-0.5,2,4.5,7,10$ ). The proposed method (SSR) is compared with a voxel-wise quantification method (AQSES) and with a quantification method dedicated to MRSI data (AQSES-MRSI). The averaged RMSE $k_{k, r}$ values of the proposed method when only the spectral term (SSR-Spectro) or only the spatial term (SSR-Spatial) are considered are also displayed. The CRLBs shown were computed from AQSES.
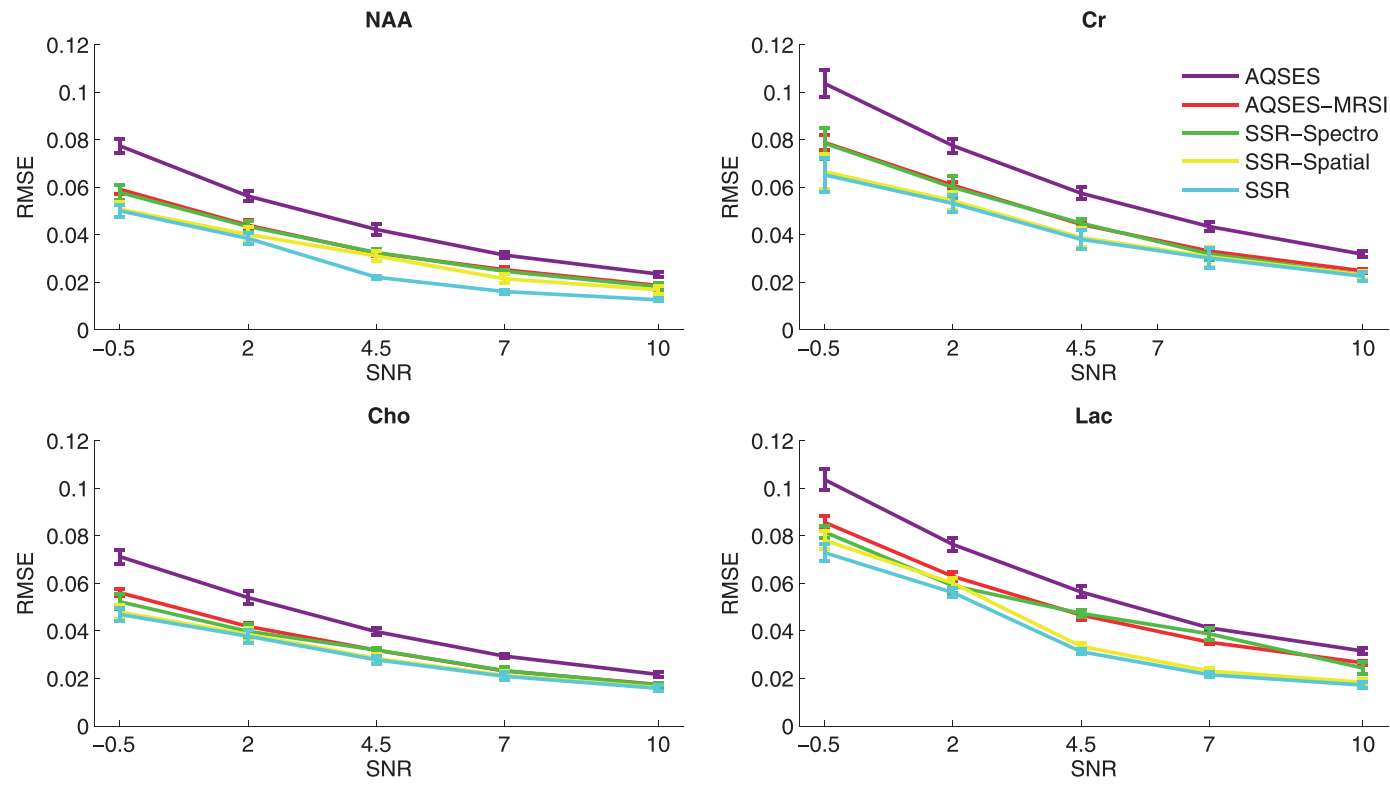

Figure 4. Mean $\mathrm{RMSE}_{k, r}$ of the estimated metabolite amplitudes averaged over all the voxels computed for 50 synthetic MRSI data sets of size $10 \times 10$ for 5 different noise levels (SNR values are: $-0.5,2,4.5,7,10$ ). The proposed method (SSR) is compared with a voxel-wise quantification method (AQSES) and with a quantification method dedicated to MRSI data (AQSES-MRSI). The averaged RMSE $k, r$ of the proposed method when only the spectral term (SSR-Spectro) or only the spatial term (SSR-Spatial) are considered are also displayed. 
volunteer and from two patients with diagnosed Glioblastoma multiforme included in a prospective clinical trial.

Note that an estimation of the noise covariance matrix $\Psi$ has been obtained from a signal (and artifact)-free part of the MRSI spectra for in vivo data. Preprocessing applied to in vivo MRSI signals includes residual water suppression (using the Hankel singular-value decomposition (HSVD) algorithm (40)), frequency correction and standard polynomial baseline correction. For the quantification of in vivo data, a metabolite basis set containing Cho, Cr, NAA and Lac was considered. The choice of this basis set was based on the fact that these metabolites are the most visible in long echo time spectra.

The robustness of the proposed method was tested on in vivo data to check whether the results are consistent with real conditions. Metabolite maps obtained using the proposed method were compared with maps obtained using voxel-wise quantification methods (AQSES (9) and QUEST (8)) and the spatially constrained approach AQSES-MRSI $(10,36))$. Regularization parameters were selected manually using a grid-search strategy as described in the subsection 'Experiments for synthetic data'. The SNR for in vivo spectra was computed in the frequency domain as $S N R=I / \sigma$, where $I$ denotes the height of the NAA peak in the real spectrum and $\sigma$ the standard deviation of the noise (measured in a signal-free region of the spectrum) (41).

\section{RESULTS}

\section{Experiments for synthetic data}

\section{Monte Carlo experiments}

Figure 3 compares the estimation error of the proposed method, referred to as spatio-spectral regularization (SSR), with the voxelwise quantification method AQSES (9) and AQSES-MRSI (10). The

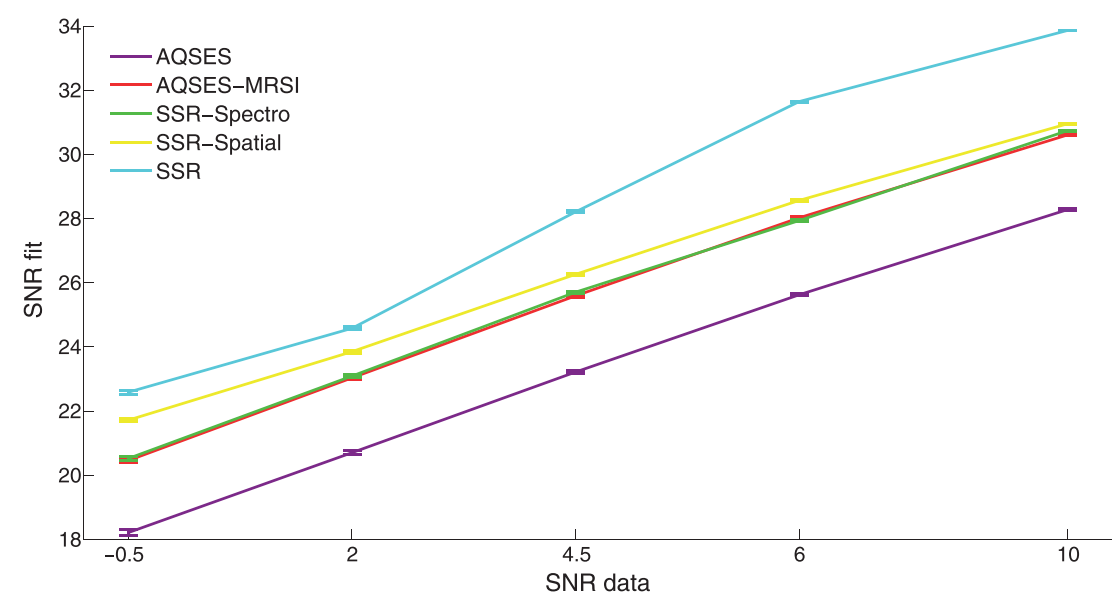

Figure 5. Mean SNR values computed for 50 synthetic MRSI data sets of size $10 \times 10$ for five different noise levels (SNR values are: $-0.5,2,4.5,7,10)$. The error bars indicate the mean $\mathrm{RMSE}_{k, r}$ of the estimated metabolite amplitudes averaged over all metabolites and all voxels.
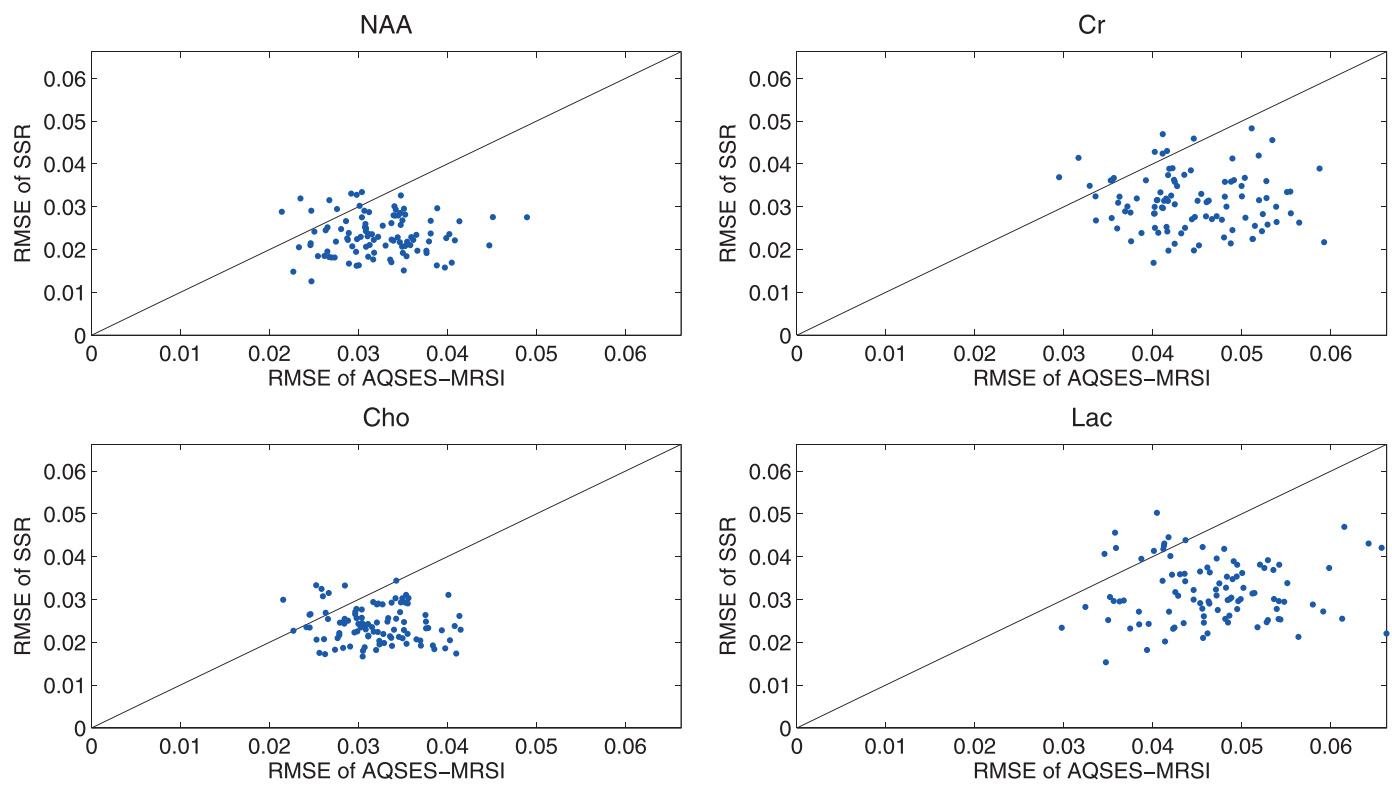

Figure 6. $\mathrm{RMSE}_{k, r}$ of the estimated metabolite amplitudes obtained with the proposed method (SSR) and with AQSES-MRSI for 50 synthetic MRSI data sets of size $10 \times 10$ at $S N R=4.5$. Each point corresponds to one voxel of the MRSI grid. 
estimation error is measured as $\mathrm{RMSE}_{k, r}$ averaged over all metabolites and all voxels. The increase in accuracy due to the incorporation of the spatial-spectral prior (in the proposed method) is clearly observed. The errors of the proposed method when only one of the regularization terms is considered, referred to as SSR-Spatial (only the spatial regularization term is used) and SSR-Spectro (only the spectral regularization term is used), are also displayed. In the same figure (Fig. 3), the errors in the metabolite amplitudes estimated with each method are also compared with the CRLB obtained from the individual voxel approach. SSR provides the best results for all noise levels, reducing the error by a mean of $41 \%$ from $24 \%$ to $54 \%$ depending on the noise level. This difference in accuracy can be observed clearly and is even below the CRLB in some cases. A similar analysis is shown in Figure 4, where the mean $\mathrm{RMSE}_{k, r}$ averaged over all voxels is shown for each individual metabolite.

Figure 5 shows the quality of the fit (measured by the SNR) for each method for different noise levels. The curves show the SNR of the fits, i.e. how close each fitted model is to the ground-truth signal. The error bars represent the mean $\mathrm{RMSE}_{k, r}$ of the estimated metabolite amplitudes averaged over all metabolites and all voxels at each noise level. Figure 6 shows the scatter plots of RMSE $\mathrm{R}_{k, r}$ of the metabolite amplitudes at each voxel. Each point corresponds to a given voxel. In most voxels, the proposed method yields lower errors for the metabolite amplitudes compared with the AQSES-MRSI method.

\section{Sharp/smooth edges}

Figure 7 presents the results obtained for a synthetic data set where the border between the regions of healthy and abnormal spectra is a sharp edge. The first row shows the ground truth for the amplitudes of NAA, Cr, Cho and Lac. The following rows show the solutions obtained from three different approaches and their differences from the ground truth. More precisely, the second row shows the results using the single-voxel approach AQSES, the third row shows the results using AQSES-MRSI, the fourth row shows the proposed spectral-spatial prior (SSR) and the last three rows show the differences between the estimated concentrations and the ground truth. The differences are measured as the percentage with respect to the maximum ground-truth value for each metabolite. The same experiment was performed for a simulated data set where the border between healthy and abnormal tissues is represented by a smoother edge. Results are shown in Figure 8.
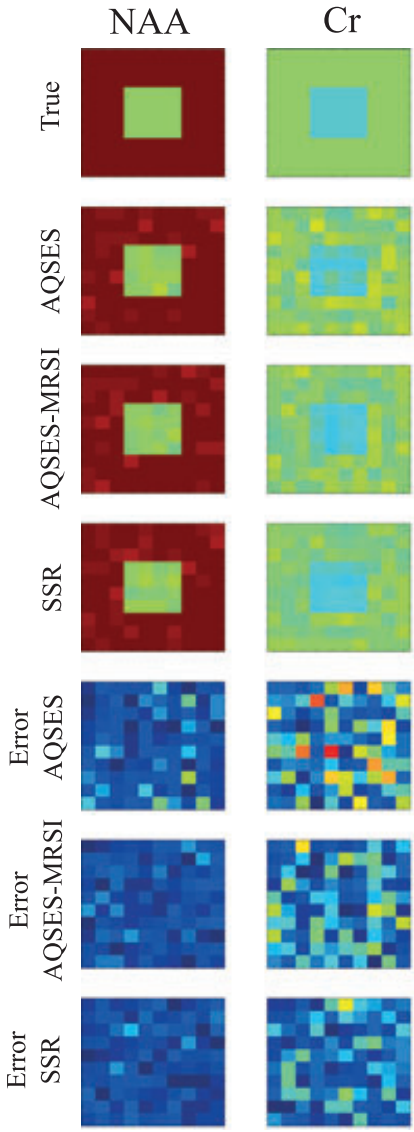
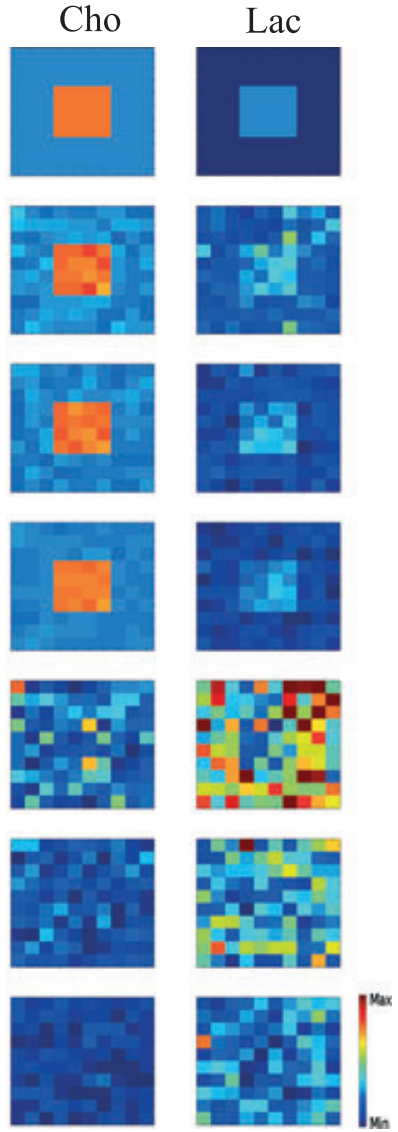

Figure 7. Synthetic MRSI data set containing two regions associated with healthy and tumour-like signals separated by a sharp edge. From top to bottom: true metabolite concentrations, estimated metabolite concentrations using AQSES, AQSES-MRSI and SSR and the difference (error) between the true metabolite concentrations and the concentrations estimated using AQSES, AQSES-MRSI and SSR. Errors are measured as a percentage with respect to the maximum ground-truth value for each metabolite.
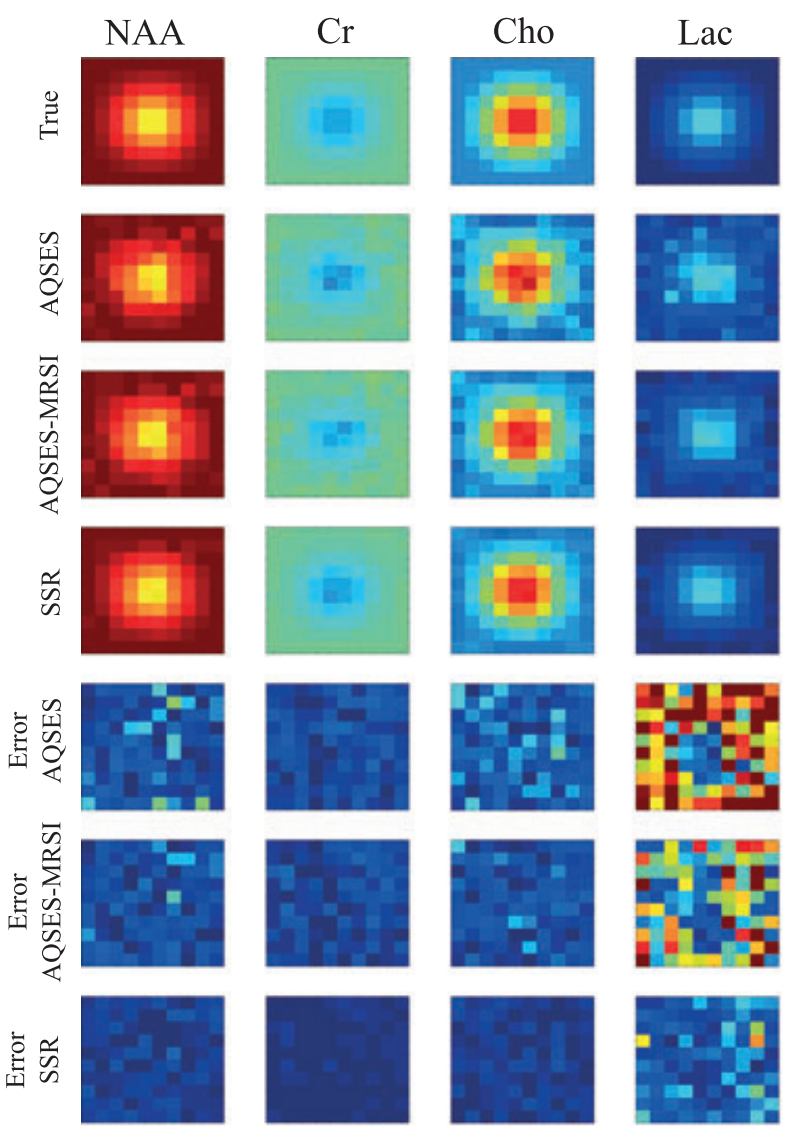

Figure 8. Synthetic MRSI data set containing a region with healthyappearing signals and a region with tumour-like signals separated by a smooth edge. From top to bottom: true metabolite concentrations, estimated metabolite concentrations using AQSES, AQSES-MRSI and SSR and the difference (error) between the true metabolite concentrations and the concentrations estimated using AQSES, AQSES-MRSI and SSR. Errors are measured as a percentage with respect to the maximum groundtruth value for each metabolite. 
Tables 1 and 2 show the SSIM values for the metabolite maps in Figures 7 and 8, respectively. These values offer an objective and quantitative measure of the accuracy of the metabolic maps obtained with the different methods.

\section{In vivo data}

We analyze the performance of our method further for in vivo MRSI data. By analyzing these maps, we can obtain a fast

Table 1. SSIM values for each metabolite amplitude map in Figure 7

\begin{tabular}{|lccc|}
\hline SSIM & AQSES & AQSES-MRSI & SSR \\
\hline NAA & 0.69 & 0.87 & 0.91 \\
Cr & 0.59 & 0.68 & 0.78 \\
Cho & 0.78 & 0.91 & 0.94 \\
Lac & 0.20 & 0.27 & 0.36 \\
\hline
\end{tabular}

Table 2. SSIM values for each metabolite amplitude map in Figure 8

\begin{tabular}{|lccc|}
\hline SSIM & AQSES & AQSES-MRSI & SSR \\
\hline NAA & 0.83 & 0.94 & 0.98 \\
Cr & 0.61 & 0.87 & 0.90 \\
Cho & 0.78 & 0.92 & 0.95 \\
Lac & 0.41 & 0.55 & 0.69 \\
\hline
\end{tabular}

visual inspection of the performance of the method. In Figure 9, the metabolite maps depict the presence of NAA, $\mathrm{Cho}$ and $\mathrm{Cr}$ as expected in healthy tissue. For the operated GBM patient (Fig. 10), the Lac map presents a spatial distribution that matches with the surgical cavity. The presence of Lac is often observed after surgery and can be linked to the postoperative reorganization of the tissue. The Cho map presents higher concentrations in correspondence with the contrastenhancing bud of the anterior part of the lesion, suggesting higher cellular proliferation in this area. For the non-operated GBM patient (Fig. 11), the NAA map presents lower concentrations spatially matching the centre of the contrastenhancement area and the necrosis that can be seen on the $T_{1}$-weighted post-gadolinium injected MR image. The Cho map shows high concentrations corresponding to the anterior left part of the contrast-enhancing lesion. The elevated presence of Cho, together with the collapse of NAA, suggests a metabolically active tumour region. The Lac map shows higher concentration in the anterior left part of the tumour, suggesting the presence of anaerobic metabolism in this area and emphasizing the heterogeneous aspect of the tumour.

Figure 12 shows nine adjacent voxels from a patient with a brain tumour and the corresponding fits from AQSES, AQSESMRSI and SSR.

Regarding the computational cost, SSR is quite efficient. The computation time depends on the size of the MRSI grid, the samples of the spectra and the level of noise. For the in vivo cases presented in this study, the computation times for processing the whole image were between 50 and $90 \mathrm{~s}$. The experiments were performed on a $64-$ bit $1.8 \mathrm{GHz}$ i7-4500U architecture with a

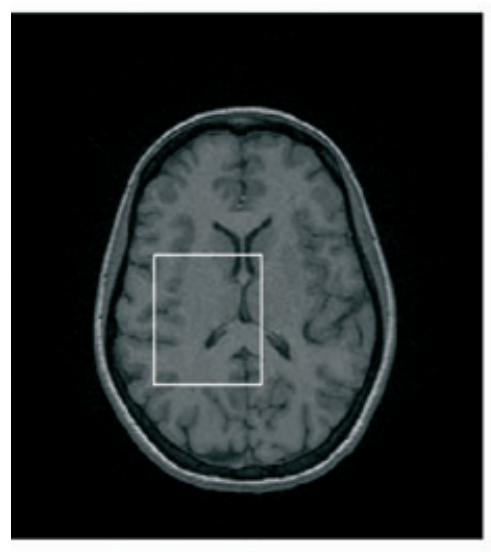

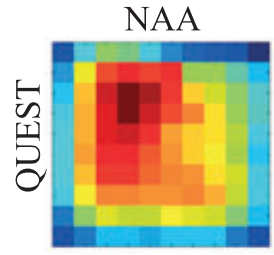

a

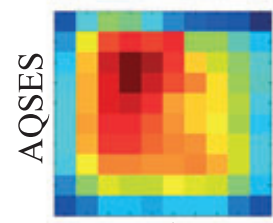

d

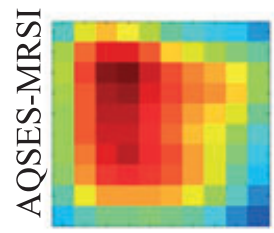

$\mathrm{g}$

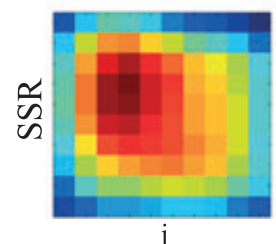

$\mathrm{Cr}$

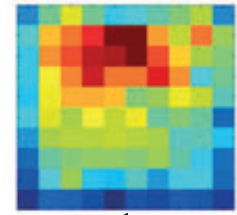

b

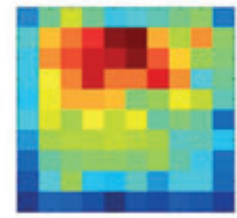

e
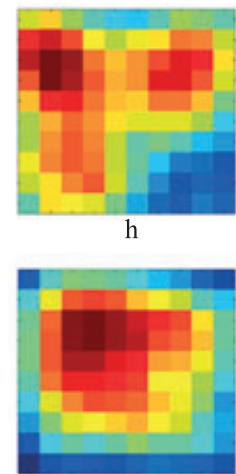

$\mathrm{k}$

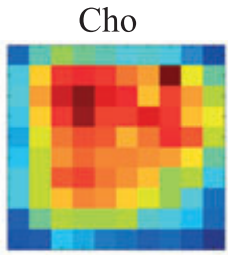

c

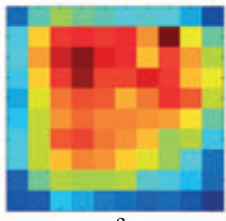

f
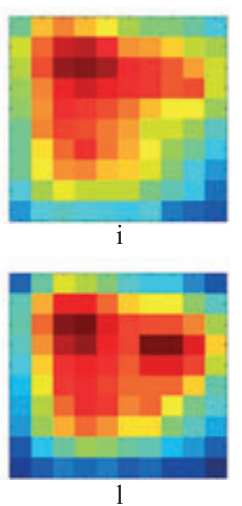

Figure 9. In vivo 3D MRSI acquisition on a healthy volunteer located in the central hemispheric right part of the brain. Metabolite maps of NAA, Cr and Cho are presented, from top to bottom respectively, using QUEST, AQSES, AQSES-MRSI and SSR. 


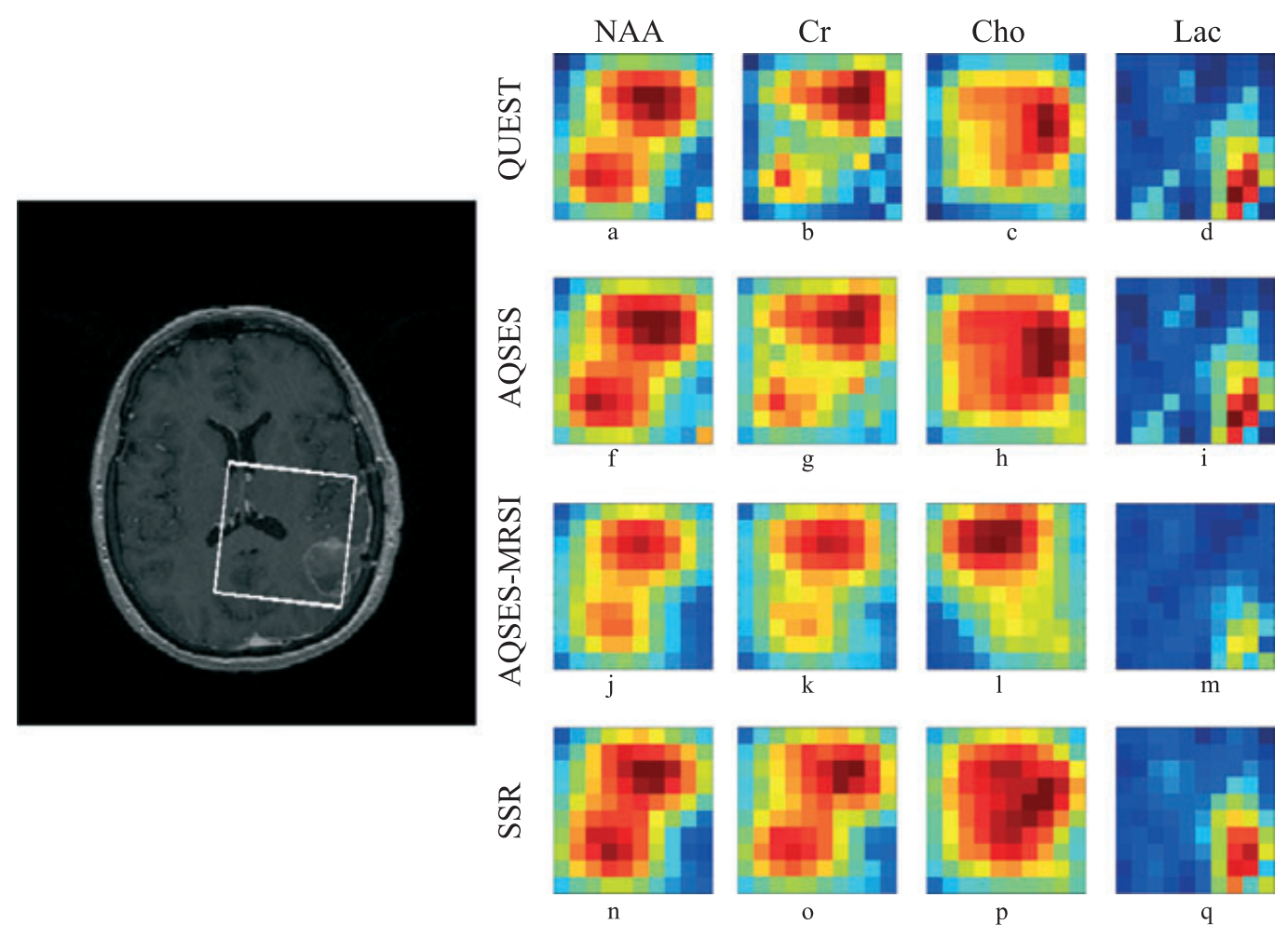

Figure 10. In vivo 3D MRSI acquisition on a resected Glioblastoma multiforme patient with a left parietal lesion associated with a thin peripheral contrast-enhancement. Metabolite maps of NAA, Cr, Cho and Lac are presented, from top to bottom respectively, using QUEST, AQSES, AQSES-MRSI and SSR.
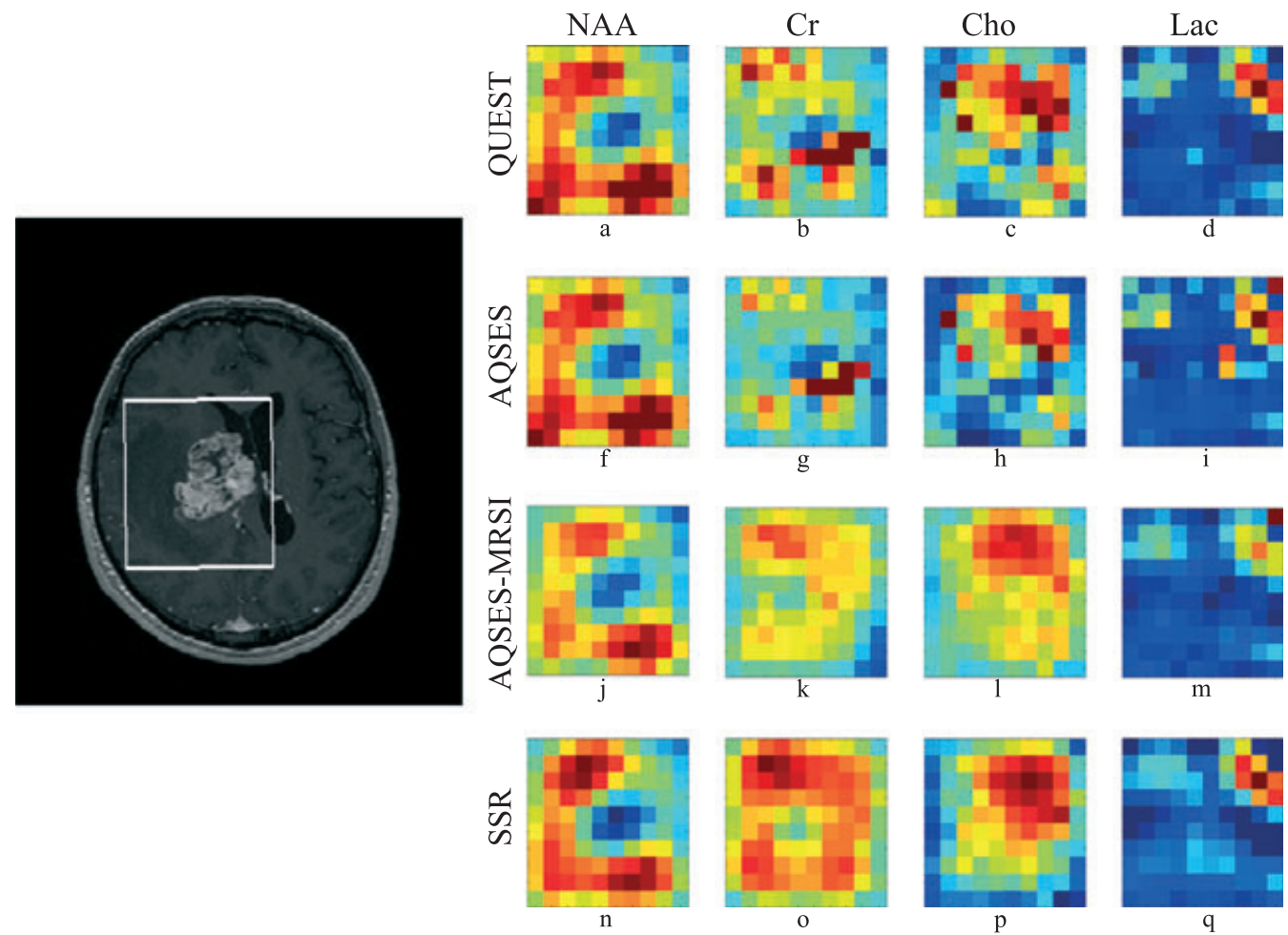

Figure 11. In vivo 3D MRSI acquisition on a biopsied Glioblastoma multiforme patient presenting a central hemispheric right lesion with a mass effect on the right lateral ventricle and a deviation of the structures through the left of the median line. Metabolite maps of NAA, Cr, Cho and Lac are presented, from top to bottom respectively, using QUEST, AQSES, AQSES-MRSI and SSR. 

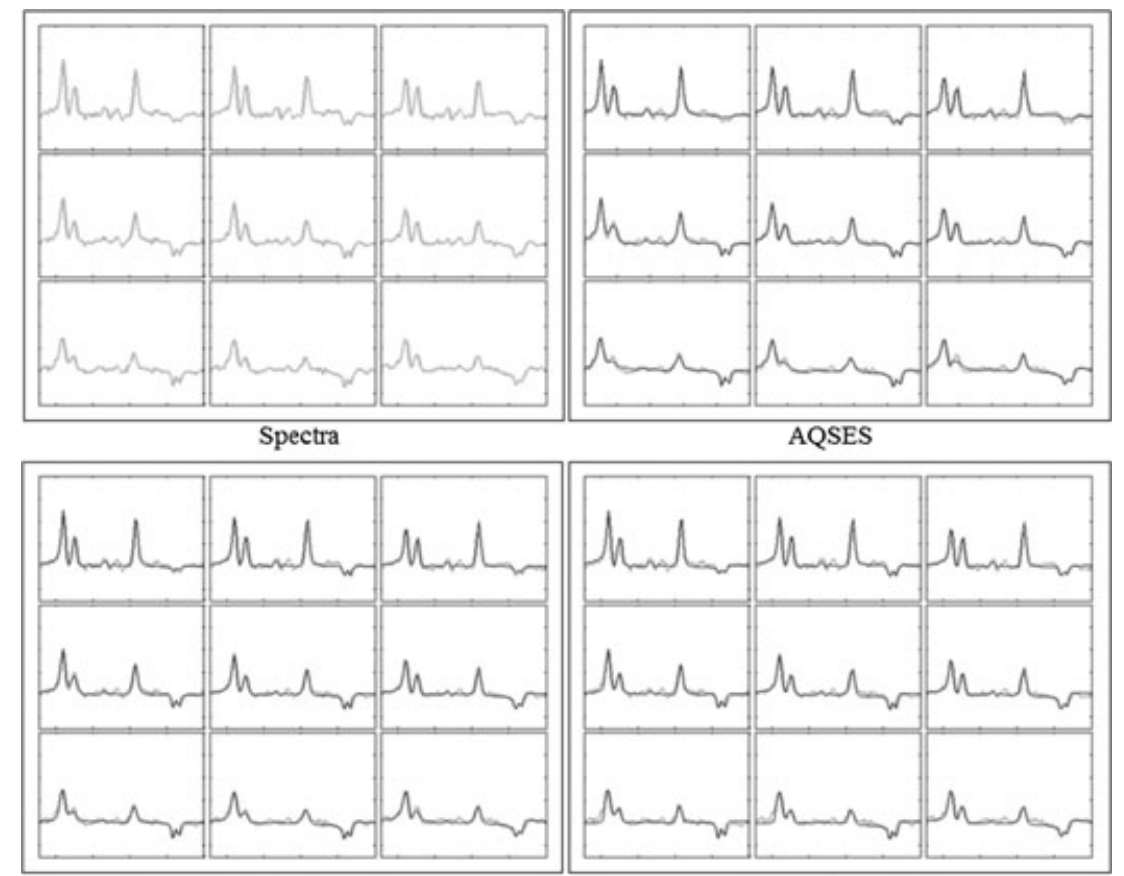

AQSES-MRSI

SSR

Figure 12. Nine adjacent voxels from a patient with a brain tumour (Gliobastoma Multiforme) and the corresponding AQSES, AQSES-MRSI and SSR fits. $\mathrm{Cr}$ and Lac peaks are erroneously fitted by the voxel-wise approach (AQSES) in many voxels. AQSES-MRSI clearly improves the quality of the fits, but $\mathrm{Cr}$ and Lac are still not well captured. SSR is able to fit all the spectra correctly.

Matlab implementation (R2013a). The convexity of the objective function guarantees convergence to a global minimum.

\section{DISCUSSION}

In order to apply the MRSI technique to routine clinical practice, it is important to maximize the accuracy and robustness of the quantification. Additionally, both processing time and technique subjectivity should be minimized by, for example, automation. The use of regularization techniques has been proved to be a powerful approach to achieve these objectives. We have presented here a quantification method based on a combined spatio-spectral regularization scheme. In contrast to previous methods, the regularization terms are expressed in the wavelet domain. The use of a combined spatio-spectral regularization scheme in a transformed domain allows the method to explore other types of prior knowledge, going beyond the conventional assumption of spatial smoothness. The benefits of imposing appropriate constraints for both spectral and spatial dimensions of MRSI data have been presented in this article.

Experiments with synthetic data show that the proposed method increases the accuracy and the robustness of the quantification against noise (Fig. 3). The presented spectral-spatial prior prevents the quantification algorithm from moving away from the true solution by narrowing down the search space. As a result, the method becomes not only more accurate but also more robust, being able to cope with signals containing high noise levels. The better accuracy provided by SSR can be explained by a more accurate fitting of the data, as shown in Figure 5. The proposed method outperforms other quantification methods even in the presence of sharp spatial features, as is shown in Figure 7 and Table 1. The higher SSIM values provided by SSR confirm objectively that the metabolic maps obtained by SSR are closer to the ground truth than the maps from AQSES and AQSES-MRSI.

Experiments with in vivo data show that the proposed method provides less noisy spatial metabolite distributions. This leads to better contoured metabolite maps. In addition, overlapping peaks (e.g. Cho and $\mathrm{Cr}$, with respective resonance frequencies at 3.2 and $3.0 \mathrm{ppm}$ ) and metabolites present at relatively low concentrations (e.g. lactate) can be better estimated (Fig. 12).

The good performance of the method can be explained by the $\ell_{1}$-norm regularization, which leads to soft thresholding of the wavelet coefficients. The wavelet thresholding acts a non-linear low-pass filter in the wavelet domain, where most image information is contained in the few largest wavelet coefficients, while the noise is uniformly spread out across all coefficients. Thus thresholding mostly affects the noise rather than the signal. The risk of missing spatial features of the smoothing filters is alleviated by wavelets, which preserve image sharpness. Another interesting property is that, even if we focus on the use of the $\ell_{1}$ norm in this manuscript, the method presented is not restricted to this case. Other $\ell_{p}$-norms (for $1 \leq p$ ) could be considered, since the method can cope with any convex penalty. Therefore, the regularization imposed on the spectral and spatial dimensions can be different. For example, it might be interesting to exploit the sparsity of the spectral dimension in combination with a spatial smoothness prior, to quantify MRSI data from a homogeneous region. It is interesting to note that the use of an averaged metabolite basis set could limit the performance of the method in the case of high variances between voxels. However, this limitation is true for any method assuming smooth spatial variations. Methods able to handle a different metabolite basis for each voxel will be analyzed in future studies. Interestingly, the proposed method requires tuning only two parameters, which give weights to the two regularization terms and can be estimated automatically from the data (39). This facilitates the 
necessary automation of the quantification process in order to make MRSI not only appealing but feasible in the clinical setting. The method presented here can be extended to incorporate 3D spatial information by using a 3D wavelet operator. This makes it especially attractive in view of the increasing interest in highresolution 3D MRSI data, where the low SNR is a main limiting factor.

\section{CONCLUSIONS}

A new method combining spatial and spectral regularizations in the wavelet domain to improve MRSI data quantification has been presented. It benefits from the combination of using a basis of metabolite profiles and incorporating spectral and spatial constraints simultaneously. In contrast to previous approaches, the suggested method combines two regularization terms to favour solutions with specific properties in both spatial and spectral dimensions. Note that the spectral parameters are not restricted to having spatially smooth variations. The method allows the use of other priors promoting properties such as the sparsity of the MRSI spectra in the wavelet domain. Experiments on synthetic data clearly demonstrated some significant improvements in quantification accuracy and robustness by using the proposed spatial-spectral regularization. Experiments on in vivo data showed well-contoured metabolite maps that can be used to define more targeted and more individualized treatments $(1,2,42)$. In addition, the presented method was able to correctly quantify metabolites that are missed by other methods due to the presence of noise or nuisance components, as has been shown for the case of lactate. This is especially relevant for the quantification of metabolites present in low concentrations. Dependence on only two hyperparameters and the use of a fast proximal algorithm that allows parallel computations facilitate the automation of the presented method, which is crucial for the acceptance of any quantification method applicable to routine clinical implementation. Future work includes the extension of the proposed method to incorporate 3D spatial information available from 3D MRSI data and validation with high spatial resolution MRSI images, where the low signal-to-noise ratio is a major limitation. The integration of an automatic hyperparameter estimation method is also an interesting prospect. Furthermore, the proposed method can be extended to incorporate more sophisticated priors, such as tissue distributions and anatomical structures extracted from companion MRI images.

\section{ACKNOWLEDGEMENTS}

This work is part of the SUMMER Marie Curie Research Training Network (PITN-GA-2011-290148), which is funded by the 7th Framework Programme of the European Commission (FP7-PEOPLE-2011-ITN). The information and views set out in this publication are those of the authors and do not necessarily reflect the official opinion of the European Union. Neither the European Union institutions and bodies nor any person acting on their behalf may be held responsible for the use that may be made of the information contained therein.

Part of this work was supported by the ANR-11-LABX-0040CIMI, in particular during the program ANR-11-IDEX-0002-02 within the thematic trimester on image processing.

\section{REFERENCES}

1. Laprie A, Catalaa I, Cassol E, McKnight TR, Berchery D, Marre D, Bachaud JM, Berry I, Moyal EC. Proton magnetic resonance spectroscopic imaging in newly diagnosed glioblastoma: predictive value for the site of postradiotherapy relapse in a prospective longitudinal study. Int. J. Radiat. Oncol. Biol. Phys. 2008; 70: 773-781.

2. Deviers A, Ken S, Filleron T, Rowland B, Laruelo A, Catalaa I, Lubrano V, Celsis P, Berry I, Mogicato G, Cohen-Jonathan Moyal E, Laprie A. Evaluation of lactate/ $\mathrm{N}$-acetyl-aspartate ratio defined with MR spectroscopic imaging before radiotherapy as a new predictive marker of the site of relapse in patients with glioblastoma multiforme. Int. J. Radiat. Oncol. Biol. Phys. 2014; 90(2): 385-393.

3. Kwock L, Smith JK, Castillo M, Ewend MG, Collichio F, Morris DE, Bouldin TW, Cush S. Clinical role of proton magnetic resonance spectroscopy in oncology: brain, breast, and prostate cancer. Lancet Oncol. 2006; 7: 859-868.

4. Posse S, Otazo R, Dager SR, Alger J. MR spectroscopic imaging: Principles and recent advances. J. Magn. Reson. Imag. 2013; 37: 1301-1325.

5. Vanhamme L, Van Den Boogaart A, Van Huffel S. Improved method for accurate and efficient quantification of MRS data with use of prior knowledge. J. Magn. Reson. 1997; 129: 35-43.

6. Soher BJ, Young K, Govindaraju V, Maudsley AA. Automated spectral analysis III: application to in vivo proton MR spectroscopy imaging. Magn. Reson. Med. 1998; 40: 822-831.

7. Provencher S. Automatic quantitation of localized in vivo ${ }^{1} \mathrm{H}$ spectra with LCModel. NMR Biomed. 2001; 14: 260-264.

8. Ratiney H, Sdika M, Coenradie Y, Cavassila S, van Ordmont D, Graveron-Demilly D. Time domain semi-parametric estimation based on a metabolite basis set. NMR Biomed. 2005; 18: 1-13.

9. Poullet JB, Sima DM, Simonetti AW, De Neuter B, Vanhamme L, Lemmerling P, Van Huffel S. An automated quantitation of short echo time MRS spectra in a open source software environment: AQSES. NMR Biomed. 2006; 20: 493-504.

10. Sava AC, Sima DM, Poullet JB, Wright AJ, Heerschap A, Van Huffel S. Exploiting spatial information to estimate metabolite levels in $2 \mathrm{D}$ MRSI of heterogeneous brain lesions. NMR Biomed. 2011; 24: 824-835.

11. Kelm BM, Kaster FO, Henning A, Weber MA, Bachert $P$, Boesiger $P$, Hamprecht FA, Menze BH. Using spatial prior knowledge in the spectral fitting of magnetic resonance spectroscopic images. NMR Biomed. 2012; 25: 1-13.

12. Boer VO, Klomp DW, Juchem C, Luijten PR, de Graaf RA. Multislice ${ }^{1} H$ MRSI of the human brain at $7 \mathrm{~T}$ using dynamic $\mathrm{B}(0)$ and $\mathrm{B}(1)$ shimming. Magn. Reson. Med. 2012; 68: 662-670.

13. Diop A, Zaim-Wadghiri Y, Briguet A, Graveron-Demilly D. Improvements of quantitation by using the Cadzow enhancement procedure prior to any linear-prediction methods. J. Magn. Reson. 1994; 105: 17-24.

14. Bao Y, Maudsley AA. Improved reconstruction for MR spectroscopic imaging. IEEE Trans. Med. Imag. 2007; 26: 686-695.

15. Nguyen HM, Haldar JP, Do MN, Zhi-Pei L. Denoising of MR spectroscopic imaging data with spatial-spectral regularization. In Proceedings of the 2010 IEEE International Symposium on Biomedical Imaging (ISBI). IEEE: Rotterdam, Netherlands; 2010: 720-723.

16. Laruelo A, Chaari L, Batatia H, Ken S, Rowland B, Laprie A, Tourneret JY. Hybrid sparse regularization for Magnetic Resonance Spectroscopy. In Proceedings of the 35th Annual International Conference of the IEEE EMBS. IEEE: Osaka, Japan; 2013: 6768-71.

17. Cavassila S, Deval S, Huegen C, van Ormondt D, Graveron-Demilly D. Cramér-Rao bounds: an evaluation tool for quantification. NMR Biomed. 2001; 14: 278-283.

18. Mierisová S, van den Boogaart A, Tkác I, Van Hecke P, Vanhamme L, Liptaj T. New approach for quantitation of short echo time in vivo ${ }^{1} \mathrm{H}$ MR spectra of brain using AMARES. NMR Biomed. 1998; 1: 32-39.

19. Smith SA, Levante TO, Meier BH, Ernst RR. Computer simulations in magnetic resonance. An object-oriented programming approach. J. Magn. Reson. 1994; 106: 75-105.

20. Graveron-Demilly D, Diop A, Briguet A, Fenet B. Product-operator algebra for strongly coupled spin systems. J. Magn. Reson. 1993; 101: 233-239.

21. Serrai H, Senhadji L, de Certaines JD, Coatrieux JL. Time-domain quanification of amplitude, chemical shift, apparent relaxation time $T_{2}$, and phase by wavelet-transform analysis: application to 
biomedical magnetic resonance spectroscopy. J. Magn. Reson. 1997; 124(1): 20-34.

22. Serrai $H$, Nadal-Desbarats L, Poptani H, Glickson JD, Senhadji L. Lactate editing and lipid suppression by continuous wavelet transform analysis: application to simulated and (1)H MRS brain tumor time-domain data. Magn. Reson. Med. 2000; 43(5): 649-656.

23. Cancino-De-Greiff HF, Ramos-Garcia R, Lorenzo-Ginori JV. Signal de-noising in magnetic resonance spectroscopy using wavelet transforms. Concepts Magn. Reson. 2002; 14(6): 388-401.

24. Zarei S, Alireazie J, Babyn P, Kassner A, Widjaja E. MRS feature extraction: time-frequency and wavelet analysis. In Proceedings of the 2nd International Conference on Bioinformatics and Biomedical Engineering ICBBE. IEEE: Shanghai, China; 2008: 1863-1866.

25. Hu S, Lustig M, Chen AP, Crane J, Kerr A, Kelley DA, Hurd R, Kurhanewicz J, Nelson SJ, Pauly JM, Vigneron DB. Compressed sensing for resolution enhancement of hyperpolarized ${ }^{1} 3 \mathrm{C}$ flyback 3DMRSI. J. Magn. Reson. 2008; 192(2): 258-264.

26. Geethanath S, Baek HM, Ganji SK, Ding Y, Maher EA, Sims RD, Choi C, Lewis MA, Kodibagkar VD. Compressive sensing could accelerate $1 \mathrm{H}$ MR metabolic imaging in the clinic. Radiol. 2012; 262(3): 985-994.

27. Vanhamme L, Sundin T, Van Hecke P, Van Huffel S. MR spectroscopy quantitation: a review of time-domain methods. NMR Biomed. 2001; 14: 233-246. DOI:10.1002/nmb.695.

28. Tikhonov A. Tikhonov regularization of incorrectly posed problems. Sov. Math. Dokl. 1963; 4: 1624-1627.

29. Ying $L, X u D$, Liang ZP. On Tikhonov regularization for image reconstruction in parallel MRI. In Proceedings of the 26th Annual International Conference of the IEEE EMBS. IEEE: San Francisco, USA; 2004: 1056-1059.

30. Guerquin-Kern M, Van De Ville D, Vonesch C, Baritaux JC, Pruessmann KP, Unser M. Wavelet regularized reconstruction for rapid MRI. IEEE Int. Symp. Biomed. Imag. (ISBI) 2009: 193-196.

31. Chaari L, Pesquet JC, Benazza-Benyahia A, Ciuciu P. A wavelet-based regularized reconstruction algorithm for SENSE parallel MRI with applications to neuroimaging. Med. Image Anal. 2011; 15(2): 185-201.

32. Chaari L, Ciuciu P, Mériaux S, Pesquet JC. Spatio-temporal wavelet regularization for parallel MRI reconstruction: application to functional MRI. Magn. Reson. Mater. Phys. Biol. Med. (MAGMA) 2014; 27 (6): 509-529. DOI:10.1007/s10334-014-0436-5.
33. Moreau JJ. Proximité et dualité dans un espace hilbertien. Bull. Soc. Math. France 1965; 93: 273-299.

34. Combettes PL, Pesquet JC. A proximal decomposition method for solving convex variational inverse problems. Inverse Probl. 2008; 24(6): (article ID: 065014).

35. Stefan D, Di Cesare F, Andrasescu A, Popa E, Lazariev A, Vescovo E, Strbak O, Williams S, Starcuk Z, Cabanas M, van Ormondt D, Graveron-Demilly D. Quantitation of magnetic resonance spectroscopy signals: the jMRUI software package. Meas. Sci. Technol. 2009; 20: 104 035-104 044. DOI:10.1088/0957-0233/20/10/104035.

36. Poullet JB, Sima DM, Luts J, Osorio Garcia M, Croitor A, Van Huffel S. Manual: Simulation Package based in In vitro Databases (SPID), 2010. URL http://homes.esat.kuleuven.be/biomed/biosource/ManualSPID. pdf. [Online; accessed 25-Feb-2015].

37. Wang Z, Bovik A, Sheikh HR, Simoncelli EP. Image quality assessment: From error visibility to structural similarity. IEEE Trans. Image Process. 2004; 13: 600-612.

38. Belge M, Kilmer ME, Miller EL. Efficient determination of multiple regularization parameters in a generalized L-curve framework. Inverse Probl. 2002; 18: 1161-1183.

39. Chaari L, Pesquet JC, Tourneret JY, Ciuciu P, Benazza-Benyahia A. A hierarchical Bayesian model for frame representation. IEEE Trans. Signal Process. 2010; 18(11): 5560-5571.

40. Laudadio T, Mastronardi N, Vanhamme L, Van Hecke P, Van Huffel S. Improved Lanczos algorithms for blackbox MRS data quantitation. J. Magn. Res. 2002; 157(2): 292-297.

41. Kreis R. Issues of spectral quality in clinical $1 \mathrm{H}$-magnetic resonance spectroscopy and a gallery of artifacts. NMR Biomed. 2004; 17(6): 361-381.

42. Ken $S$, Vieillevigne $L$, Franceries $X$, Simon $L$, Supper $C$, Lotterie JA, Filleron T, Lubrano V, Berry I, Cassol E, Delannes M, Celsis P, Moyal Cohen-Jonathan E, Laprie A. Integration method of 3D MR spectroscopy into treatment planning system for glioblastoma IMRT dose painting with integrated simultaneous boost. Radiat. Oncol. 2013; 8: 1.

43. Combettes PL, Pesquet JC. Proximal splitting methods in signal processing. In Fixed-Point Algorithms for Inverse Problems in Science and Engineering, Bauschke HH, Burachik R, Combettes PL, Elser V, Luke DR, Wolkowicz H (eds). Springer: New York, 2011, pp. 185-212. 


\section{APPENDIX}

\section{OPTIMIZATION PROCEDURE}

This appendix details the optimization procedure used to minimize the criterion in (9). The algorithm used for the minimization is the simultaneous direction method of multipliers (34) and is based on the concept of proximity operators (33). In what follows, we recall the definition of a proximity operator and provide the expressions for the proximity operators involved in our quantification problem. Definition 1

(33) Let $\Gamma_{0}(\chi)$ be the class of proper lower semicontinous convex functions from a separable real Hilbert space $\chi$ to ] $\left.-\infty,+\infty\right]$ and let $\phi \in \Gamma_{0}(\chi)$. For every $\mathrm{x} \in \chi$, the function $\phi+\|\cdot-\mathrm{x}\|^{2} / 2$ achieves its infimum at a unique point denoted by prox ${ }_{\phi} \mathrm{x}$. The operator $\operatorname{prox}_{\phi}: \chi \rightarrow \chi$ is the proximity operator of $\phi$.

In this work, as the observed data are complex-valued, the definition of proximity operators is extended to a class of convex functions defined for complex-valued variables. For the function

$$
\left.\left.\Phi: \mathbb{C}^{R} \rightarrow\right]-\infty,+\infty\right] x \mapsto \varphi^{\operatorname{Re}}[\operatorname{Re}(x)]+\varphi^{\operatorname{Im}}[\operatorname{Im}(x)]
$$

where $\varphi^{\mathrm{Re}}$ and $\varphi^{\operatorname{Im}}$ are functions in $\Gamma_{0}\left(\mathbb{R}^{R}\right)$ and $\operatorname{Re}(x)$ (respectively $\left.\operatorname{Im}(x)\right)$ is the vector of the real parts (respectively imaginary parts) of the component of $x \in R$, the proximity operator is defined as

$$
\operatorname{prox\Phi }: \mathbb{C}^{R} \rightarrow{ }^{R} X \mapsto \operatorname{prox}_{\Phi^{\mathrm{Re}}}[\operatorname{Re}(x)]+\operatorname{prox}_{\Phi^{\operatorname{lm}}}[\operatorname{Im}(x)]
$$

\section{Proximity operator of the data fidelity term}

Based on the above definitions, and according to standard rules about the calculation of proximity operators ((43), Table 1), the proximity operator of the data fidelity term $\mathcal{J}_{\text {wLS }}$ is given by

$$
\operatorname{prox}_{\mathcal{J}_{\mathrm{WLS}}} \boldsymbol{A}=\left(\boldsymbol{I}_{d}+2 \Psi^{-1}\right)^{-1}\left(\boldsymbol{H A}+2 \Psi^{-1} \boldsymbol{S}\right)
$$

\section{Proximity operators of the regularization terms}

Regarding the regularization terms, the proximity operator of $\Phi_{\mu, \alpha}^{p}$ for every $\xi \in \mathbb{C}$ is given by

$$
\operatorname{prox}_{\Phi_{\mu, \alpha}^{p}} \xi=\operatorname{sign}(\xi) \eta
$$

where $\eta=\eta^{\mathrm{Re}}+i \eta^{\mathrm{Im}}$ is the unique solution in $\mathbb{C}_{+}$to $\eta+p \eta^{p-1} \alpha=|\xi-\mu|$. If $p=1$, this proximity operator simplifies as follows:

$$
\begin{aligned}
\operatorname{prox}_{\Phi_{\mu, \alpha}^{1}} \xi= & \operatorname{sign}[\operatorname{Re}(\xi-\mu)] \max \{|\operatorname{Re}(\xi-\mu)|-\operatorname{Re}(\alpha), 0\} \\
& +i \operatorname{sign}[\operatorname{Im}(\xi-\mu)] \max \{|\operatorname{Im}(\xi-\mu)|-\operatorname{Im}(\alpha), 0\}
\end{aligned}
$$

\begin{tabular}{|c|c|}
\hline \multicolumn{2}{|c|}{ Algorithm 1 Simultaneous direction method of multipliers } \\
\hline \multicolumn{2}{|c|}{ Set $\gamma>0, \varepsilon \in] 0,1\left[, \boldsymbol{Y}_{i, 0} \in M \times R, \boldsymbol{Z}_{i, 0} \in{ }^{M \times R}, i \in\{1, \ldots, N\}, \boldsymbol{Q}=\sum_{n=1}^{N} \boldsymbol{H}^{*} \boldsymbol{H}(N=3\right.$ in our case). Set $n=1$. } \\
\hline 1: & repeat $\quad N$ \\
\hline 2: & Set $\boldsymbol{U}_{n}=\boldsymbol{Q}^{-1} \sum \boldsymbol{H}^{*}\left(\boldsymbol{Y}_{i, n}-\boldsymbol{Z}_{i, n}\right)$ \\
\hline 3: & for $i=1$ to $N$ वर् \\
\hline 4: & Set $\boldsymbol{S}_{i, n}=\boldsymbol{H} \boldsymbol{A}_{n}$ \\
\hline 5: & Calculate $\boldsymbol{Y}_{i, n+1}=\operatorname{prox}_{\gamma g_{i}}\left(\boldsymbol{S}_{i, n}+\boldsymbol{Z}_{i, n}\right)$ \\
\hline 6: & Set $\boldsymbol{Z}_{i, n+1}=\boldsymbol{Z}_{i, n}+\boldsymbol{S}_{i, n}-\boldsymbol{Y}_{i, n+1}$ \\
\hline 7: & end for \\
\hline 8: & $n \leftarrow n+1$ \\
\hline 9: & until $\left|\mathcal{J}\left(\boldsymbol{U}_{n}\right)-\mathcal{J}\left(\boldsymbol{U}_{n-1}\right)\right|<\varepsilon$ \\
\hline 10: & return $\hat{\boldsymbol{U}}=\hat{U}_{n}$ \\
\hline
\end{tabular}

By referring to the spatial and spectral regularization terms $g_{1}$ and $g_{2}$ in (7) and (8), it turns out that the proximity operator of the regularization term can be obtained by calculating the proximity operator of $\Phi_{\mu, \alpha}^{p}{ }^{\circ}{ }^{\top}$ and $\Phi_{\mu, \alpha}^{p}{ }^{\circ} F$. These operators can be calculated based on ((34), Lemma 2.4), leading to $\operatorname{prox}_{\Phi_{\mu, \alpha}^{p} \circ T}=T^{-1} \operatorname{oprox}_{\Phi_{\mu, \alpha}^{p}} \circ T$ (similarly for prox $\Phi_{\mu, \alpha}^{p} \circ$ ).

Based on the calculated proximity operators, the resulting algorithm for the minimization of the optimality criterion in (9) is described below in Algorithm 1.

It is important to note that the computations in this algorithm can be performed in a parallel manner (steps 4-6 can be computed in parallel for each i). In addition, the global convergence of the algorithm to an optimal solution to the minimization problem is guaranteed. The parameter $\gamma$ is related to the convergence speed of the algorithm and has been chosen by cross-validation for each case (34). The stopping parameter $\epsilon$ has been set to $10^{-6}$ and the algorithm normally converges in fewer than 50 iterations. 